\title{
Human Macrophage Activation \\ Modulation of Mannosyl, Fucosyl Receptor Activity In Vitro by Lymphokines, Gamma and Alpha Interferons, and Dexamethasone
}

Taole Mokoena and Siamon Gordon

Sir William Dunn School of Pathology, University of Oxford, South Parks Road, Oxford OXI 3RE, United Kingdom

\begin{abstract}
We describe a sensitive assay to measure immune activation of human macrophages in cell culture. Freshly isolated blood monocytes from normal subjects lack the ability to endocytose and degrade mannosyl-terminated glycoconjugates via specific receptors, but acquired this activity after cultivation in autologous serum for $\sim 3$ d. Addition of specific antigen, purified protein derivative, or $\mathbf{T}$ cell mitogens to mononuclear cells prevented the appearance of macrophage mannosyl receptor activity and lymphokine, $\gamma$-, and $\alpha$-interferons selectively downregulated receptor activity in monocyte-macrophage targets. The effects of antigen challenge and $\boldsymbol{\gamma}$-interferon on mannosyl receptors can be prevented by $10^{-8} \mathrm{M}$ dexamethasone. Dexamethasone also inhibited release of another macrophage activation marker, plasminogen activator, which was increased by both $\boldsymbol{\gamma}$ - and $\alpha$-interferons. These studies show that activation of human macrophages is regulated by opposing actions of lymphokines and glucocorticoids.
\end{abstract}

\section{Introduction}

Activated macrophages $(\mathrm{M} \phi)^{1}$ play an important role in immunity to various antigens and intracellular pathogens, in part because of an enhanced capacity to generate toxic metabolites of oxygen (1). Lymphokine products of antigen-challenged $\mathrm{T}$ lymphocytes activate $\mathbf{M} \phi$ by inducing selective changes in $\mathbf{M} \phi$ plasma membrane and secretory properties (2). Recent studies in several laboratories indicate that $\gamma$-interferon (Ifn) accounts for much of $\mathrm{M} \phi$ activation by lymphokines (3-5).

We know little about the nature and control of $\mathrm{M} \phi$ activation in man, and its role in immunopathology. Tissue $\mathrm{M} \phi$ are difficult to obtain and characterize in patients, and circulating blood monocytes may not reflect the activity of cells in the periphery. Previous studies with mouse peritoneal $\mathbf{M} \phi$ have shown that a $\mathbf{M} \phi$-restricted lectinlike receptor for mannosyl, fucosyl-terminated glycoproteins (MFR) is selectively

Dr. Mokoena is a Nuffield Dominion Scholar. Address correspondence and reprint requests to Dr. Gordon.

Received for publication 23 April 1984 and in revised form 19 September 1984.

1. Abbreviations used in this paper: HMP, hexose monophosphate; Ifn, interferon(s); $\mathbf{M} \phi$, macrophage; Man-BSA, mannose 59-bovine serum albumin; MFR, M $\phi$ receptor for mannosyl, fucosyl-terminated glycoconjugates; MNC, mononuclear cell(s); NSE, nonspecific esterase; PMA, phorbol myristate acetate; PA, plasminogen activator; PPD, purified protein derivative; $R B$, respiratory burst.

J. Clin. Invest.

(c) The American Society for Clinical Investigation, Inc. 0021-9738/85/02/0624/08 \$1.00

Volume 75, February 1985, 624-631 down-regulated by lymphokine treatment (6). We show here that human blood monocytes acquire MFR activity during maturation in culture and that antigen challenge in the presence of specifically sensitized lymphocytes prevents its appearance on $\mathbf{M} \phi$. Both $\gamma$ - and $\alpha$-Ifn are able to down-regulate the MFR in monocyte/M $\mathbf{M}$ targets. Dexamethasone, an anti-inflammatory corticoid, modulates MFR activity by opposing the activation of $\mathrm{M} \phi$ by lymphokines.

\section{Methods}

Media. Culture medium consisted of RPMI 1640 (Gibco-Biocult, Paisley, Scotland) supplemented with $2 \mathrm{mM}$ glutamine, $20 \mathrm{mM}$ Hepes, $20 \mu \mathrm{g} / \mathrm{ml}$ gentamycin, and $10 \% \mathrm{vol} / \mathrm{vol}$ autologous serum unless stated otherwise. Heat inactivation of serum $\left(56^{\circ} \mathrm{C} / 30 \mathrm{~min}\right)$ reduced $\mathrm{M} \phi$ MFR activity by $10-20 \%$ and was not done routinely. Homologous human, foetal bovine, newborn calf, and horse serum were not optimal for development of MFR by human monocytes.

Reagents were obtained as follows: purified protein derivative (PPD, Connaught Labs, Toronto, Canada), phytohemagglutinin (Difco Labs, Surrey, United Kingdom), concanavalin A (Sigma, Poole, Dorset, United Kingdom), pokeweed mitogen (Gibco-Biocult), and lipopolysaccharide (Difco Labs). Recombinant human interferon-gamma (Ifn$\gamma$, [7]), lot 1323-10, produced by Genentech, Inc., was supplied by Boehringer Ingelheim, Vienna, Austria. The material was supplied $>97 \%$ pure on SDS-polyacrylamide gel electrophoresis, specific activity $1.2 \times 10^{7} \mathrm{U} / \mathrm{mg}$, in an antiviral bioassay, pyrogen $<0.5 \mathrm{ng} / \mathrm{ml}$. Human lymphoblastoid interferon (Ifn- $\alpha$ ), batch $785 / 3$, was generously provided by Dr. K. Fantes, Wellcome Research Laboratories, Beckenham, Kent, United Kingdom (8). This material was a mixture of eight major and some minor components, contained no Ifn- $\gamma$, and was supplied $\sim 50 \%$ pure, specific activity $7.1 \times 10^{7} \mathrm{U} / \mathrm{mg}$ protein, before addition of human plasma albumin as stabilizer. Ifn preparations were stored as small aliquots at $-70^{\circ} \mathrm{C}$, thawed once, and used immediately or within a week of storage at $4^{\circ} \mathrm{C}$. Relative antiviral activity was confirmed by Dr. J. Cardosa, University of Oxford, using human fibroblasts and West Nile virus. Dr. Fantes also supplied specific anti-Ifn- $\alpha$ antibodies (9), bovine antiserum (batch 931/6.1.83) and bovine globulin IMP 245 , antiviral neutralizing activities, $\sim 1.7 \times 10^{6}$ and $\sim 3 \times 10^{6} \mathrm{U} / \mathrm{ml}$, respectively, as well as control bovine serum. Mannose 59-bovine serum albumin (Man-BSA), galactose 55-BSA, fucose 73-BSA, and $N$-acetyl-glucosamine BSA were provided by $\mathrm{Dr}$. P. Stahl, Washington University, St. Louis, MO. (10). Man-BSA was radioiodinated by a chloramine $\mathrm{T}$ method (11) which yielded $3 \mu \mathrm{Ci} /$ $\mu \mathrm{g}$ protein, of which $80-90 \%$ was precipitable by $10 \%$ TCA. Dexamethasone (Sigma) was dissolved in dimethylsulfoxide at $10^{-2} \mathrm{M}$, stored in aliquots at $-20^{\circ} \mathrm{C}$, and diluted in $\mathrm{Ca}^{++}$and $\mathrm{Mg}^{++}$free phosphate-buffered saline (PBS, Oxoid, Basingstoke, Hampshire, United Kingdom) before use. Yeast mannan and zymosan type I were obtained from Sigma. Human plasminogen and ${ }^{125} \mathrm{I}$-labeled fibrin coated 24-well trays were prepared as described (12). Radioisotopes (Na ${ }^{125} \mathrm{I},\left[6-{ }^{3} \mathrm{H}\right]$ thymidine and $\left.\mathrm{D}\left[1-{ }^{14} \mathrm{C}\right] \mathrm{glucose}\right)$ were from Amersham International, Amersham, Bucks, United Kingdom.

Cells. Healthy adult volunteers were bled at the Oxford Regional Blood Transfusion Service $(450 \mathrm{ml}$, in citrate phosphate dextrose) or at the laboratory $(50-100 \mathrm{ml}$, in acid citrate dextrose). $20-50 \mathrm{ml}$ clotted blood was used to prepare autologous serum. Mononuclear cell 
(MNC) fractions were prepared within $2 \mathrm{~h}$ by Ficoll-Hypaque density gradient centrifugation (Pharmacia, Hounslow, Middlesex, United Kingdom). Yields were $6-13 \times 10^{5} \mathrm{MNC} / \mathrm{ml}$ blood, of which $\sim 20 \%$ were monocytes by nonspecific esterase, type I, staining (13). For some experiments, monocytes were purified by adherence of MNC populations to gelatin-coated flasks (14) in complete medium (90 min, $37^{\circ} \mathrm{C}$ ). Nonadherent cells, mainly lymphocytes, were washed off with warm RPMI 1640; adherent monocytes, 13-26\% of total, $>90 \%$ nonspecific esterase $+v e$, were detached by vigorous shaking in ice-cold $\mathrm{Ca}^{++}$- and $\mathrm{Mg}^{++}$-free PBS. Monocytes detached more readily after overnight incubation. Total MNC or adherent monocytes were cultivated in complete medium at $37^{\circ} \mathrm{C}$ in the presence of $5 \% \mathrm{CO}_{2}$ either in flat bottom 96-well plates (Sterilin, Teddington, Middlesex, United Kingdom) at $2 \times 10^{5} / 0.2 \mathrm{ml} /$ well, in 24-well Linbro trays at $2 \times 10^{6} / 1 \mathrm{ml} /$ well, or in T25 flasks (Nunclon, Gibco-Biocult) at $16-20 \times 10^{6} / 5 \mathrm{ml}$. Cultures were refed by replacing half of the medium at day $3 / 4$ and then once weekly, as necessary. Cell morphology and viability were monitored by phase-contrast microscopy.

Lymphokine. Tuberculin skin testing was kindly performed by Dr. Juel-Jensen, Radcliffe Infirmary, Oxford. MNC cultures, $2 \times 10^{6} / \mathrm{ml}$, from Mantoux-positive donors were treated with 5-10 $\mu \mathrm{g} / \mathrm{ml} \mathrm{PPD} \mathrm{in}$ complete medium for $3 \mathrm{~d}$. The supernatant was cleared by centrifugation, filter sterilized, and aliquots stored at $-20^{\circ} \mathrm{C}$ were stable for at least 6 mo. Control lymphokine was prepared by adding PPD at the end of the period of culture.

Assays. Mannosyl-fucosyl receptor activity was assayed as described (15). In brief, degradation of ${ }^{125}$ I-Man-BSA was determined in the absence and presence of mannan. Cells in 96-well trays were routinely incubated with $\sim 100 \mathrm{ng}{ }^{125}$ I-Man-BSA, three wells without and two with mannan, $1.25 \mathrm{mg} / \mathrm{ml}$, usually for $2 \mathrm{~d}$. Aliquots of medium (20 $\mu \mathrm{l}$ out of $200 \mu \mathrm{l})$ were assayed for TCA soluble radioactivity. Results were expressed as the mean of mannan-inhibitable degradation per 20$\mu l$ sample. Wells without cells were included in each assay and showed $<10 \%$ degradation. Specific degradation (10-90\%) was dependent on the concentration of ${ }^{125} \mathrm{I}-\mathrm{Man}-\mathrm{BSA}$ in the range $25-250 \mathrm{ng} /$ culture, the number of adherent monocytes plated $\left(2 \times 10^{4}-2 \times 10^{5} /\right.$ well $)$, and the duration of cultivation. Nonspecific degradation by control cells was $<2 \%$ of total.

Specific uptake by MFR was measured by incubating adherent cells with $200 \mathrm{ng}{ }^{125} \mathrm{I}$-Man-BSA in serum-free RPMI 1640 , with or without $1.25 \mathrm{mg} / \mathrm{ml}$ mannan for $30 \mathrm{~min}$ at $37^{\circ} \mathrm{C}$, and measuring cellassociated radioactivity. Lymphocyte proliferation was measured by 4 $\mathrm{h}$ incorporation of $\left[{ }^{3} \mathrm{H}\right]$ thymidine $(1 \mu \mathrm{Ci} /$ well $)$ after cultivating $\mathrm{MNC}$ for 3-4 d (16). Results were expressed as the mean of triplicate cultures or as a proliferation index (see below). Respiratory burst (RB) activity was measured by assay of hexose monophosphate (HMP) shunt activity as described (17). Plasminogen activator (PA) release and lysozyme secretion were measured as described (12). HLA-A, B and C antigens were determined by the Oxford Regional Blood Transfusion Service.

\section{Results}

Monocytes express MFR activity after maturation in culture. Freshly isolated blood monocytes did not degrade ${ }^{125} \mathrm{I}-\mathrm{Man}$ BSA, but acquired this activity after $3 \mathrm{~d}$ cultivation in autologous serum (Fig. $1 A$ ), in parallel with morphologic evidence of $\mathrm{M} \phi$ maturation. Similar results have been reported by Shepherd and her colleagues (18). Acquisition of MFR activity was optimal at $10-20 \%$ autologous serum (Fig. $1 B$ ), in parallel with maturation. The appearance of MFR after a lag period was highly reproducible for a particular donor, although individual donors varied in the precise time of onset, 3-4 d, and up to fourfold in the extent of degradation. Control experiments, not shown, confirmed that degradation was due to $\mathbf{M} \phi$, which was $>95 \%$ pure, and not lymphocytes, and was similar in the presence or absence of unstimulated lymphocytes. Monocytes also failed to bind or internalize ${ }^{125}$ I-Man-BSA until $>3 \mathrm{~d}$ of cultivation. Specificity of the receptor was confirmed by neoglycoprotein inhibitors of uptake of ${ }^{125} \mathrm{I}$-Man-BSA in the order fucose 73-BSA-Man-BSA $>N$-acetyl-glucosamine BSA $\gg$ galactose 55-BSA (18).

Antigen- and mitogen-stimulated lymphocytes decrease $M \phi$ $M F R$. Addition of PPD to MNC cultures derived from tuberculin-sensitive individuals markedly reduced subsequent MFR activity (Fig. $1 A$, Table I). Inhibition of MFR activity by PPD was associated with positive skin sensitivity, aggregation of MNC in culture (not shown), and increased incorporation of

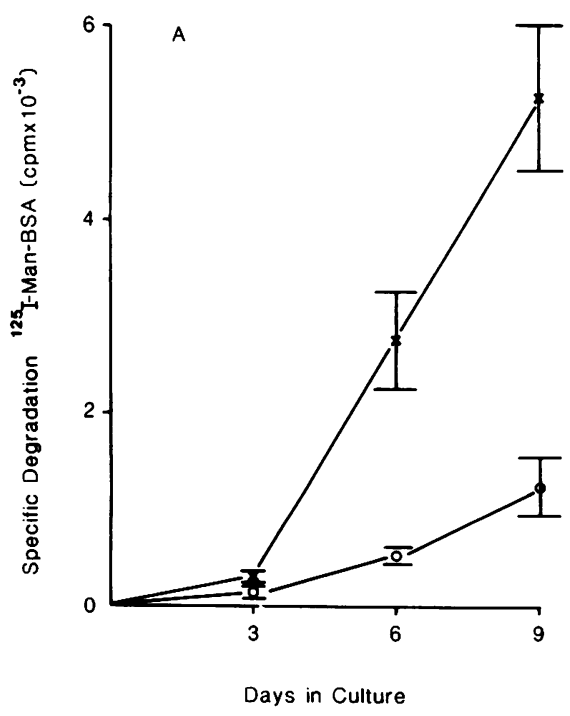

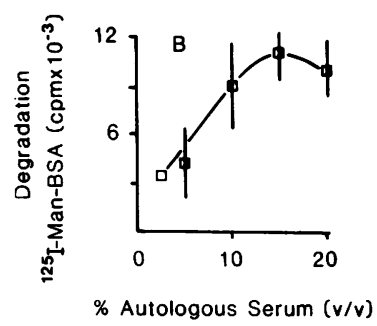

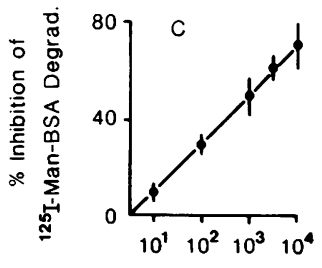

PPD $\mathrm{ng} / \mathrm{ml}$
Figure 1. MFR activity of human monocytes in culture. $(A)$ Effect of antigen on appearance of MFR. $2 \times 10^{5}$ total mononuclear cells (MNC) from tuberculin-positive donors were maintained in culture with $(O)$ or without $(X)$ PPD, $10 \mu \mathrm{g} / \mathrm{ml}$ final, in microtitre wells containing RPMI 1640 supplemented with 10\% autologous serum. 7-d cultures were refed by half medium replacement on day 4 with no further antigen addition. Cultures were pulsed with ${ }^{125} \mathrm{I}$-Man-BSA $3 \mathrm{~d}$ before assay on the day indicated. Pooled results from experiments with four separate donors. $(B)$ Role of serum. $2 \times 10^{5}$ adherent monocyte/M $\phi$ were cultured in RPMI supplemented with various concentrations of autologous serum for $7 \mathrm{~d}$. Cultures were refed by half medium replacement on day 4 and pulsed with ${ }^{125}$ I-Man-BSA $2 \mathrm{~d}$ before assay. Results were pooled from experiments with two separate donors. $(C)$ Antigen dose response. $2 \times 10^{5}$ total $\mathrm{MNC}$ from tuberculin-positive donors were cultured in the presence of different concentrations of PPD for 6-9 d. Cultures were refed by $1 / 2$ medium replacement without further PPD addition on day 4 and pulsed with ${ }^{125} \mathrm{I}$-Man-BSA $2 \mathrm{~d}$ before assay. Results were pooled from experiments with four separate donors. 
Table I. Effect of PPD on MFR Activity and Lymphocyte Proliferation in Total Mononuclear Cultures from Tuberculin Positive and Negative Individuals

\begin{tabular}{|c|c|c|c|c|c|c|c|}
\hline \multirow[b]{2}{*}{ Donor } & \multirow[b]{2}{*}{$\begin{array}{l}\text { Tuberculin } \\
\text { skin test }\end{array}$} & \multicolumn{3}{|c|}{ Mannan-specific degradation of ${ }^{125} \mathrm{I}$-Man-BSA $\left(\mathrm{cpm} \times 10^{-3}\right)^{*}$} & \multicolumn{3}{|c|}{$\left[{ }^{3} \mathrm{H}\right]$ thymidine incorporation $\left(\mathrm{cpm} \times 10^{-3}\right) \ddagger$} \\
\hline & & Control & $\begin{array}{l}+\mathrm{PPD} \\
10 \mu \mathrm{g} / \mathrm{ml}\end{array}$ & $\S \%$ inhibition & Control & $\begin{array}{l}+ \text { PPD } \\
10 \mu \mathrm{g} / \mathrm{ml}\end{array}$ & "PI \\
\hline T.M. & $+\mathrm{ve}$ & $6.48 \pm 0.21$ & $1.44 \pm 0.1$ & 78 & $2.02 \pm 0.22$ & $22.7 \pm 5.2$ & 10 \\
\hline L.T. & $+\mathrm{ve}$ & $5.02 \pm 0.60$ & $1.66 \pm 0.001$ & 67 & $1.4 \pm 0.79$ & $27.9 \pm 9.9$ & 19 \\
\hline S.H.L. & $+\mathrm{ve}$ & $2.3 \pm 0.34$ & $1.05 \pm 0.27$ & 55 & $2.12 \pm 0.55$ & $8.89 \pm 4.9$ & 3.2 \\
\hline M.W. & $+\mathrm{ve}$ & $11.7 \pm 4.1$ & $1.89 \pm 0.33$ & 84 & $0.82 \pm 0.44$ & $2.07 \pm 0.54$ & 1.5 \\
\hline B.R. & -ve & $5.78 \pm 0.25$ & $5.68 \pm 0.38$ & 2 & $6.13 \pm 0.48$ & $7.05 \pm 1.4$ & 0.15 \\
\hline A.H. & $-v e$ & $6.92 \pm 0.74$ & $6.43 \pm 0.8$ & 7 & $1.6 \pm 0.33$ & $1.34 \pm 0.29$ & -0.16 \\
\hline J.H. & $-v e$ & $11.0 \pm 1.5$ & $11.8 \pm 2.0$ & -7 & $1.43 \pm 0.95$ & $1.85 \pm 0.15$ & 0.30 \\
\hline A.T. & $-v e$ & $4.50 \pm 0.81$ & $4.37 \pm 0.09$ & 3 & ND & & \\
\hline
\end{tabular}

* $2 \times 10^{5}$ total MNC culture \pm PPD for 6-7 d were pulsed with ${ }^{125}$ I-Man-BSA \pm Mannan $2 \mathrm{~d}$ before assay. $¥ 2 \times 10^{5}$ total MNC cultured $\pm \mathrm{PPD}$ for 3-4 $\mathrm{d}$ were pulsed with $\left[{ }^{3} \mathrm{H}\right]$ thymidine $4 \mathrm{~h}$ before harvesting. $\$$ Percent inhibition $=($ Control $-\mathrm{PPD}$ treated $) /(\mathrm{Control}) \times 100$. " PI $($ proliferation index $)=($ PPD treated-control $) /($ Control $)$.

$\left[{ }^{3} \mathrm{H}\right]$ thymidine by lymphocytes, although not necessarily to the same extent. Sensitized lymphocytes were required to induce the change in MFR activity by antigen; PPD had no effect when added to thoroughly washed adherent monocytes (not shown). Decreased expression of MFR was not due to loss of M $\phi$ viability, since lysozyme secretion was unimpaired (not shown) and cells excluded trypan blue. Inhibition of MFR was proportional to log PPD concentration (Fig. $1 C$ ), could be demonstrated in sensitized individuals $3 \mathrm{~d}$ after adding 1-10 $\mu \mathrm{g} / \mathrm{ml}$ PPD to freshly explanted mononuclear cultures, and ranged between 60 and $90 \% ; 50 \%$ inhibition was observed $2 \mathrm{~d}$ after adding PPD to MNC which had been cultivated for up to $4 \mathrm{~d}$. The effect of PPD on MFR was prolonged up to 9-11 $\mathrm{d}$, and could be reversed by washing off nonadherent cells and cultivating adherent $\mathbf{M} \phi$ further without antigen. Addition of the $\mathrm{T}$ lymphocyte mitogens concanavalin $\mathrm{A}$ and phytohemagglutinin to MNC cultures from randomly selected donors decreased M $\phi$ MFR activity, unlike the $B$ cell mitogens pokeweed mitogen and lipopolysaccharide $(<30 \%$ change, not shown).

Down regulation of $M \phi M F R$ by lymphokine and interferon. Lymphokines obtained by PPD challenge of tuberculin-sensitive individuals suppressed MFR activity of adherent $\mathbf{M} \dot{\phi}$ targets prepared from the same or different donors by $20-80 \%$, depending on the individual tested and the concentration of lymphokine (not shown). Active and control lymphokines were assayed on targets from tuberculin-negative donors to avoid stimulation of residual lymphocytes by antigen. To extend the range of donor target, we tested homogeneous lymphokines, defined Ifn preparations on M $\phi$ MFR activity, and also determined the HLA-ABC phenotype of donors as a possible correlate of individual variation. Both $\gamma$ - and $\alpha$-Ifn were potent inhibitors of M $\phi$ MFR activity, whether assayed on total MNC targets (Table II A) or on adherent monocytes/

Table II A. Effect of $\gamma$ - and $\alpha$-Ifn on MFR Activity of Total MNC Cultures

\begin{tabular}{|c|c|c|c|c|c|c|c|c|c|c|}
\hline \multirow[b]{3}{*}{ Donor } & \multirow[b]{3}{*}{ Ifn } & \multirow{3}{*}{$\begin{array}{l}\text { Day of } \\
\text { assay }\end{array}$} & \multicolumn{7}{|c|}{ Mannan specific ${ }^{125}$ I-Man-BSA degradation } & \multirow[b]{3}{*}{ HLA phenotype } \\
\hline & & & \multirow{2}{*}{$\begin{array}{l}\text { Control } \\
\left(\mathrm{cpm} \times 10^{-3}\right)\end{array}$} & \multicolumn{2}{|l|}{$5 \mathrm{U} /$ well Ifn } & \multicolumn{2}{|l|}{$50 \mathrm{U} /$ well Ifn } & \multicolumn{2}{|l|}{$500 \mathrm{U} /$ well Ifn } & \\
\hline & & & & $\mathrm{cpm} \times 10^{-3}$ & $\%$ Inhibition & $\mathrm{cpm} \times 10^{-3}$ & \% Inhibition & $\mathrm{cpm} \times 10^{-3}$ & $\%$ Inhibition & \\
\hline G.P. & $\gamma$ & 5 & $10.5 \pm 0.51$ & $4.92 \pm 0.54$ & 53 & $4.48 \pm 1.0$ & 54 & $0.96 \pm 0.29$ & 91 & $\mathrm{~A} 3,-, \mathrm{B} 7, \mathrm{BW} 14$ \\
\hline R.Br. & $\gamma$ & 14 & $10.4 \pm 3.0$ & $10.8 \pm 2.5$ & -4 & $1.43 \pm 1.6$ & 86 & $0.48 \pm 0.46$ & 95 & ND \\
\hline S.H.L. & $\gamma$ & 21 & $3.18 \pm 1.2$ & $2.12 \pm 0.29$ & 32 & $0.76 \pm 0.2$ & 76 & ND & & $\begin{array}{l}\text { A2 B17(16) C3? AW33 } \\
\text { DR3 DR6 BW4 }\end{array}$ \\
\hline V.C. & $\gamma$ & 6 & $9.86 \pm 1.3$ & ND & & $1.45 \pm 0.46$ & 85 & ND & & $\mathrm{A} 2,-, \mathrm{B} 8,-$ \\
\hline D.A. & $\gamma$ & 8 & $8.33 \pm 2.2$ & ND & & $5.48 \pm 1.3$ & 35 & ND & & $\mathrm{A} 1 ; \mathrm{A} 21 ; \mathrm{B} 8, \mathrm{~B} 14 \mathrm{CW} 7$ \\
\hline J.P. & $\gamma$ & 8 & $13.0 \pm 1.8$ & ND & & $1.43 \pm 0.81$ & 89 & ND & & $\mathrm{A} 2,-, \mathrm{B} 7, \mathrm{~B} 40$ \\
\hline J.S. & $\gamma$ & 9 & $14.5 \pm 2.4$ & ND & & $3.81 \pm 1.7$ & 74 & ND & & A9, A28, B7, B14 \\
\hline G.P. & $\alpha$ & 6 & $10.7 \pm 0.25$ & $3.82 \pm 0.44$ & 64 & $1.06 \pm 0.19$ & 90 & $0.54 \pm 0.09$ & 95 & See above \\
\hline A.L.W. & $\alpha$ & 6 & $23.7 \pm 4.9$ & $9.12 \pm 3.3$ & 62 & $2.84 \pm 0.46$ & 88 & $2.1 \pm 0.42$ & 91 & ND \\
\hline R.Br. & $\alpha$ & 14 & $10.4 \pm 3.0$ & $10.6 \pm 2.45$ & -1 & $5.53 \pm 0.39$ & 47 & $1.50 \pm 0.86$ & 86 & ND \\
\hline S.H.L. & $\alpha$ & 21 & $3.18 \pm 1.2$ & $1.37 \pm 0.37$ & 57 & $0.60 \pm 0.10$ & 81 & ND & & See above \\
\hline D.A. & $\alpha$ & 8 & $8.33 \pm 2.2$ & ND & & $3.31 \pm 1.33$ & 60 & ND & & See above \\
\hline J.P. & $\alpha$ & 8 & $13.0 \pm 1.8$ & ND & & $5.46 \pm 0.74$ & 58 & ND & & See above \\
\hline J.S. & $\alpha$ & 9 & $14.5 \pm 2.4$ & ND & & $5.24 \pm 1.5$ & 64 & ND & & See above \\
\hline
\end{tabular}

Legend: see bottom of Table II B. 
Table II B. Effect of $\gamma$ - and $\alpha$-Ifn on MFR Activity of Adherent Monocyte/M $\phi$ Cultures

\begin{tabular}{|c|c|c|c|c|c|c|c|c|c|c|}
\hline \multirow[b]{3}{*}{ Donor } & \multirow[b]{3}{*}{ Ifn } & \multirow{3}{*}{$\begin{array}{l}\text { Day of } \\
\text { assay }\end{array}$} & \multicolumn{7}{|c|}{ Mannan specific ${ }^{125} \mathrm{I}$-Man-BSA degradation } & \multirow[b]{3}{*}{ HLA phenotype } \\
\hline & & & \multirow{2}{*}{$\begin{array}{l}\text { Control } \\
\left(\mathrm{cpm} \times 10^{-3}\right)\end{array}$} & \multicolumn{2}{|l|}{$5 \mathrm{U} /$ well Ifn } & \multicolumn{2}{|l|}{$50 \mathrm{U} /$ well Ifn } & \multicolumn{2}{|c|}{$500 \mathrm{U} /$ well Ifn } & \\
\hline & & & & $\mathrm{cpm} \times 10^{-3}$ & \% Inhibition & $\mathrm{cpm} \times 10^{-3}$ & \% Inhibition & $\mathrm{cpm} \times 10^{-3}$ & \% Inhibition & \\
\hline G.P. & $\gamma$ & 5 & $39.9 \pm 4.5$ & $9.04 \pm 2.9$ & 77 & $8.88 \pm 0.56$ & 78 & $1.7 \pm 0.19$ & 96 & $\mathrm{~A} 3,-, \mathrm{B} 7$ \\
\hline G.P. & $\gamma$ & 9 & $23.3 \pm 1.4$ & $1.46 \pm 0.13$ & 94 & $0.50 \pm 0.13$ & 98 & $1.01 \pm 0.19$ & 91 & $\mathrm{~A} 3,-, \mathrm{B} 7$ \\
\hline S.M.H. & $\gamma$ & 9 & $18.4 \pm 1.6$ & $2.46 \pm 3.3$ & 83 & $0.33 \pm 0.37$ & 98 & ND & & $\begin{array}{l}\text { A1; A11, B8, BW38 } \\
\text { (BW16)? CW7 }\end{array}$ \\
\hline T.S. & $\gamma$ & 7 & $4.42 \pm 0.56$ & $4.60 \pm 0.35$ & -4 & ND & & ND & & $\begin{array}{l}\text { A28, AW30, B12 } \\
\text { (BW44) } \\
\text { B13, CW5, CW6 }\end{array}$ \\
\hline J.Cr. & $\gamma$ & 8 & $24.6 \pm 4.9$ & ND & & $14.02 \pm 3.8$ & 43 & ND & & $\mathrm{A} 1 ;-, \mathrm{B} 8, \mathrm{~B} 40$ \\
\hline A.B. & $\gamma$ & 8 & $36.1 \pm 4.0$ & ND & & $18.01 \pm 3.6$ & 50 & ND & & $\mathrm{A} 2,-, \mathrm{B} 12, \mathrm{BW} 35$ \\
\hline D.A. & $\gamma$ & 8 & $10.4 \pm 1.7$ & ND & & $1.83 \pm 0.66$ & 82 & ND & & $\mathrm{A} 2,-, \mathrm{B} 7, \mathrm{~B} 40$ \\
\hline M.W. & $\gamma$ & 9 & $19.5 \pm 1.3$ & ND & & $12.2 \pm 2.1$ & 39 & ND & & $\mathrm{A} 2, \mathrm{AW} 22, \mathrm{~B} 14, \mathrm{~B} 40$ \\
\hline G.P. & $\alpha$ & 5 & $39.9 \pm 4.5$ & $12.5 \pm 0.61$ & 69 & $7.15 \pm 0.81$ & 82 & $4.92 \pm 0.49$ & 88 & See above \\
\hline G.P. & $\alpha$ & 9 & $23.3 \pm 1.4$ & $2.0 \pm 0.24$ & 91 & $2.58 \pm 0.64$ & 85 & $0.30 \pm 0.17$ & 98 & See above \\
\hline T.S. & $\alpha$ & 7 & $4.42 \pm 0.56$ & $2.41 \pm 0.48$ & 45 & ND & & ND & & See above \\
\hline M.F. & $\alpha$ & 6 & $8.25 \pm 1.5$ & ND & & $2.78 \pm 0.28$ & 66 & $3.42 \pm 0.43$ & 59 & ND \\
\hline J.Cr. & $\alpha$ & 8 & $24.6 \pm 4.9$ & ND & & $12.7 \pm 2.6$ & 48 & ND & & See above \\
\hline A.B. & $\alpha$ & 8 & $36.1 \pm 4.0$ & ND & & $25.0 \pm 4.2$ & 32 & ND & & See above \\
\hline D.A. & $\alpha$ & 8 & $10.4 \pm 1.7$ & ND & & $1.83 \pm 0.6$ & 82 & ND & & See above \\
\hline M.W. & $\alpha$ & 9 & $19.5 \pm 1.3$ & ND & & $12.3 \pm 5.1$ & 37 & ND & & See above \\
\hline J.D. & $\alpha$ & 10 & $20.3 \pm 3.9$ & ND & & $16.5 \pm 0.61$ & 19 & ND & & $\mathrm{A} 2, \mathrm{~A} 3, \mathrm{~B} 7, \mathrm{~B} 15, \mathrm{CW} 3$ \\
\hline
\end{tabular}

$2 \times 10^{5}$ total MNC (Table IIA) or $2 \times 10^{5}$ adherent monocyte/M $\phi$ (Table IIB) were cultured in the presence of $\gamma$ - or $\alpha$-Ifn. ${ }^{125}$ I-Man-BSA \pm mannan were added $2 \mathrm{~d}$ before assay. Neither Ifn was effective at $0.5 \mathrm{U} /$ well, while at concentrations $>500 \mathrm{U}$ there was no further increase in effect. See Tables III and IV for assays of donors such as T.S. at concentrations between 50 and $200 \mathrm{U} /$ well. ND, not determined.

M $\phi$ (Table II B). The extent of inhibition depended on the dose of Ifn and the individual, and $50-90 \%$ inhibition could be elicited in most subjects by $5-50 \mathrm{U} /$ culture. Occasional donors were relatively resistant to $50 \mathrm{U} /$ culture. Individuals varied in their sensitivity to either Ifn, irrespective of their HLA-ABC phenotype. Cell targets were sensitive to Ifn added at the onset of culture or later, treatments which reduced MFR activity to a comparable level (not shown). Interferon action was manifest within 2-3 d, depending on the control level of MFR activity, and suppression could be maintained for up to $21 \mathrm{~d}$ in the continued presence of Ifn. The effect on MFR activity was reversible, $50-100 \%, 3 \mathrm{~d}$ after washout of Ifn (not shown).

Table III shows that inhibition of M $\phi$ MFR by $\alpha$-Ifn could be substantially prevented by specific anti- $\alpha$-Ifn antibodies which did not block the effect of $\gamma$-Ifn. The antibodies and sera did not influence MFR activity directly (not shown). Further control experiments showed that Ifn-treated $\mathbf{M} \phi$ were

Table III. Specific Antibody Blocks Effect of $\alpha$-Ifn on MRF

\begin{tabular}{|c|c|c|c|c|c|c|c|c|c|}
\hline \multirow[b]{2}{*}{ Donor } & \multicolumn{2}{|c|}{ Untreated control $\alpha$-Ifn } & \multirow[b]{2}{*}{$\%$ Control } & \multicolumn{2}{|c|}{$\alpha$-Ifn + Anti- $\alpha$-Ifn globulin } & \multicolumn{2}{|c|}{$\alpha$-Ifn + anti- $\alpha$-Ifn serum } & \multicolumn{2}{|c|}{$\alpha$-Ifn + control serum } \\
\hline & $\mathrm{cpm} \times 10^{-3}$ & $\mathrm{cpm} \times 10^{-3}$ & & $\mathrm{cpm} \times 10^{-3}$ & $\%$ Control & $\mathrm{cpm} \times 10^{-3}$ & $\%$ Control & $\mathrm{cpm} \times 10^{-3}$ & $\%$ Control \\
\hline $\mathrm{Cr}$ & 24.59 & 12.71 & 52 & 22.18 & 90 & 19.22 & 78 & 7.92 & 32 \\
\hline A.B. experiment 1 & 27.44 & 17.64 & 64 & 25.29 & 92 & 17.99 & 66 & ND & \\
\hline A.B. experiment 2 & 36.06 & 24.92 & 69 & ND & & 29.35 & 81 & ND & \\
\hline \multirow[t]{2}{*}{ T.S. } & 18.66 & 2.32 & 13 & 18.74 & 103 & 14.56 & 80 & 2.06 & 11 \\
\hline & & $\gamma$-Ifn & & \multicolumn{2}{|c|}{$\underline{\gamma-I f n}+$ anti- $\alpha$-Ifn globulin } & \multicolumn{2}{|c|}{$\underline{\gamma-I f n+\text { anti- } \alpha \text {-Ifn serum }}$} & \multicolumn{2}{|c|}{$\boldsymbol{\gamma}$-Ifn + control serum } \\
\hline $\mathrm{Cr}$ & 24.59 & 14.02 & 57 & ND & & ND & & ND & \\
\hline A.B. experiment 2 & 36.06 & 18.01 & 50 & 15.02 & 42 & 19.44 & 54 & 14.49 & 40 \\
\hline T.S. & 18.16 & 3.54 & 20 & 0.30 & 2 & 1.36 & 8 & 1.68 & 9 \\
\hline
\end{tabular}

$2 \times 10^{5}$ adherent monocytes/M $\phi$ were treated with $50 \mathrm{U} /$ well of either $\gamma$ - or $\alpha$-IFN in presence of anti-human $\alpha$-Ifn bovine globulin or antihuman $\alpha$-Ifn bovine serum at neutralizing doses determined by preliminary experiments. Normal bovine serum with no specific anti- $\alpha$-IFN activity was used in parallel. Data show 2-d mannan specific ${ }^{125}$ I-Man-BSA degradation on 7/8-d cultures. ND, not done. 
fully viable and secreted similar levels of lysozyme to untreated cells (not shown). Morphologic effects of $\gamma$-Ifn on M $\phi$ were similar to those observed after lymphokine. The $\mathbf{M} \phi$ became more spindly after 4 to $7 \mathrm{~d}$ than untreated cells in autologous serum. Monocytes treated with $\alpha$-Ifn were clustered, more rounded, and less adherent than $\gamma$-Ifn-treated or control cells.

Dexamethasone counteracts action of lymphokine and interferon on M申 MFR. Addition of dexamethasone $\left(10^{-9}-10^{-8}\right.$ M) to control total mononuclear or adherent monocyte/M $\phi$ cultures had either no effect or enhanced MFR activity up to 2-3-fold (Table IV, A-C). Dexamethasone effectively and reproducibly prevented the decrease of MFR activity induced by PPD challenge or $\gamma$-Ifn, whereas the effect on $\alpha$-Ifn action was inconsistent. A fixed concentration of dexamethasone, $10^{-8} \mathrm{M}$, could be partially counteracted by raising the concentration of $\gamma$-Ifn severalfold. Increasing the concentration of dexamethasone did not improve protection against either interferon (not shown). In total mononuclear cultures challenged with PPD, addition of dexamethasone could be delayed $4 \mathrm{~d}$ and still effectively prevent MFR decrease (not shown). Dexamethasone prevented the effect of $\boldsymbol{\gamma}$-Ifn on adherent targets free of lymphocytes, $<5 \%$ (Table IV and not shown), which was evidence that dexamethasone influenced lymphokine action on $\mathbf{M} \phi$ rather than its production.

Modulation of monocyte fibrinolysis and respiratory burst activity. The effects of interferons and dexamethasone were next extended to other markers of macrophage activation. Table $\mathrm{V}$ shows that purified monocytes/M $\phi$ displayed substantial fibrinolytic activity when cultivated on ${ }^{125}$ I-fibrin in autologous serum for 1-3 d. This activity was shown to be dependent on the presence of plasminogen (not shown). Fibrinolysis was enhanced by relatively low concentrations of recombinent

Table IV. Effect of Dexamethasone on MFR

Modulation by Antigen and Ifn;

(IV A) PPD Challenge of Tuberculin-positive Individuals

\begin{tabular}{|c|c|c|c|c|c|}
\hline \multirow[b]{2}{*}{ PPD } & & \multicolumn{2}{|c|}{ No dexamethasone } & \multicolumn{2}{|c|}{$10^{-8} \mathrm{M}$ Dexamethasone } \\
\hline & & $\mathrm{cpm} \times 10^{-3}$ & $\%$ Control & $\mathrm{cpm} \times 10^{-3}$ & $\%$ Control \\
\hline \multicolumn{6}{|l|}{$\mu g / m l$} \\
\hline \multirow[t]{4}{*}{ M.J. (d8) } & 0 & $11.9 \pm 2.7$ & 100 & $11.8 \pm 0.38$ & 99 \\
\hline & 0.1 & $4.33 \pm 0.29$ & 36 & $9.27 \pm 1.10$ & 78 \\
\hline & 1.0 & $6.91 \pm 0.89$ & 51 & $10.7 \pm 1.43$ & 90 \\
\hline & 10 & $4.30 \pm 0.50$ & 36 & $10.4 \pm 2.54$ & 87 \\
\hline \multirow[t]{4}{*}{ S.W. (d6) } & 0 & $15.9 \pm 1.6$ & 100 & $17.8 \pm 4.30$ & 112 \\
\hline & 0.1 & $5.81 \pm 2.3$ & 37 & $16.3 \pm 2.95$ & 103 \\
\hline & 1.0 & $6.63 \pm 1.1$ & 42 & $16.5 \pm 2.64$ & 104 \\
\hline & 10 & $5.30 \pm 0.87$ & 33 & $16.3 \pm 3.2$ & 102 \\
\hline \multirow[t]{2}{*}{ D.H. (d4) } & 0 & 1.13 & 100 & 1.38 & 123 \\
\hline & 5 & 0.72 & 64 & 1.60 & 142 \\
\hline \multirow[t]{2}{*}{ A.E. (d5) } & 0 & $1.89 \pm 0.10$ & 100 & $6.36 \pm 0.33$ & 337 \\
\hline & 10 & $0.41 \pm 0.06$ & 22 & $2.61 \pm 0.04$ & 138 \\
\hline
\end{tabular}

$2 \times 10^{5}$ total MNC from tuberculin-positive donors were incubated with different PPD concentrations and with or without dexamethasone for indicated duration. If assayed after $>5 \mathrm{~d}$, then the cultures were refed by $1 / 2$ medium change and addition of more dexamethasone but not PPD. Data represent mean of specific degradation of

${ }^{125}$ I-Man-BSA measured over $2 \mathrm{~d}$.
Table IV B. $\gamma$-Ifn

\begin{tabular}{|c|c|c|c|c|c|}
\hline \multirow{2}{*}{\multicolumn{2}{|c|}{ Ifn (U/well) }} & \multicolumn{2}{|c|}{ No dexamethasone } & \multicolumn{2}{|c|}{$10^{-8} \mathrm{M}$ Dexamethasone } \\
\hline & & \multirow{2}{*}{$\frac{\mathrm{cpm} \times 10^{-3}}{10.0 \pm 1.90}$} & \multirow{2}{*}{$\begin{array}{l}\text { \% Control } \\
100\end{array}$} & \multirow{2}{*}{$\frac{\mathrm{cpm} \times 10^{-3}}{9.88 \pm 1.16}$} & \multirow{2}{*}{$\begin{array}{c}\text { \% Contro } \\
99\end{array}$} \\
\hline T.S. & 0 & & & & \\
\hline & 25 & $2.01 \pm 0.92$ & 20 & $10.6 \pm 2.19$ & 106 \\
\hline & 50 & $1.54 \pm 0.88$ & 15 & $8.46 \pm 0.67$ & 84 \\
\hline & 100 & $2.10 \pm 0.19$ & 21 & $6.38 \pm 0.21$ & 64 \\
\hline & 200 & $1.70 \pm 0.11$ & 17 & $5.96 \pm 0.88$ & 60 \\
\hline \multirow[t]{5}{*}{ M.A.D. } & 0 & $31.7 \pm 3.71$ & 100 & $31.1 \pm 2.46$ & 98 \\
\hline & 25 & $7.80 \pm 1.78$ & 25 & $16.7 \pm 1.98$ & 53 \\
\hline & 50 & $7.74 \pm 1.14$ & 24 & $15.3 \pm 0.80$ & 48 \\
\hline & 100 & $6.78 \pm 0.39$ & 21 & $16.4 \pm 2.03$ & 52 \\
\hline & 200 & $4.63 \pm 1.35$ & 15 & $12.7 \pm 2.27$ & 40 \\
\hline \multirow[t]{4}{*}{ V.C. } & 0 & $9.6 \pm 1.26$ & 100 & $14.4 \pm 2.11$ & 146 \\
\hline & 50 & $1.45 \pm 0.40$ & 15 & $13.0 \pm 3.47$ & 132 \\
\hline & 100 & $1.90 \pm 0.40$ & 19 & $8.13 \pm 2.79$ & 82 \\
\hline & 200 & $1.11 \pm 0.41$ & 11 & $5.60 \pm 1.22$ & 57 \\
\hline \multirow[t]{2}{*}{ J.P. } & 0 & $12.4 \pm 1.70$ & 100 & $21.90 \pm 0.39$ & 177 \\
\hline & 50 & $1.10 \pm 0.08$ & 9 & $2.60 \pm 1.53$ & 21 \\
\hline \multirow[t]{3}{*}{ J.P. } & 0 & $23.2 \pm 4.8$ & 100 & $20.4 \pm 1.55$ & 88 \\
\hline & 50 & $2.71 \pm 1.42$ & 12 & $15.8 \pm 0.87$ & 68 \\
\hline & 100 & ND & & $9.25 \pm 1.44$ & 40 \\
\hline
\end{tabular}

Legend: see bottom Table IV C.

$\gamma$-Ifn. Dexamethasone opposed this action depending on the relative concentration of the two reagents, and also inhibited basal activity in some individuals. In other experiments (not

Table IV C. $\alpha-I f n$

\begin{tabular}{lcccccc}
\hline & & \multicolumn{2}{l}{ No dexamethasone } & & \multicolumn{2}{c}{$10^{-8} \mathrm{M}$ Dexamethasone } \\
\cline { 7 - 7 } \cline { 6 - 7 } Ifn (U/well) & $\mathrm{cpm} \times 10^{-3}$ & \% Control & & $\mathrm{cpm} \times 10^{-3}$ & \% Control \\
\hline T.S. & 0 & $8.54 \pm 1.01$ & 100 & & $9.76 \pm 1.68$ & 114 \\
& 25 & $1.91 \pm 0.59$ & 22 & & $1.91 \pm 0.99$ & 22 \\
& 50 & $1.96 \pm 0.82$ & 23 & & $2.45 \pm 0.21$ & 29 \\
& 100 & $1.86 \pm 0.66$ & 22 & & $2.27 \pm 0.60$ & 27 \\
& 200 & $2.02 \pm 0.06$ & 24 & & $1.77 \pm 0.85$ & 21 \\
M.A.D. & 0 & $24.0 \pm 1.68$ & 100 & & $24.6 \pm 1.80$ & 102 \\
& 25 & $19.9 \pm 2.10$ & 83 & & $22.2 \pm 2.80$ & 92 \\
& 50 & $17.7 \pm 0.88$ & 73 & & $24.5 \pm 2.62$ & 102 \\
& 100 & $19.5 \pm 2.78$ & 81 & & $21.1 \pm 3.33$ & 88 \\
& 200 & $12.4 \pm 0.94$ & 47 & & $14.4 \pm 4.18$ & 60 \\
J.H. & 0 & $9.92 \pm 0.45$ & 100 & & $11.1 \pm 1.23$ & 112 \\
& 2.5 & $4.20 \pm 1.02$ & 42 & & $9.69 \pm 0.94$ & 98 \\
& 5 & $5.06 \pm 0.81$ & 51 & & $7.76 \pm 0.99$ & 78 \\
& 25 & $5.77 \pm 1.75$ & 58 & & $10.4 \pm 0.67$ & 105 \\
& 50 & $3.42 \pm 0.39$ & 34 & & $10.0 \pm 0.51$ & 101 \\
J.P. & 0 & $12.4 \pm 1.70$ & 100 & & $21.9 \pm 0.39$ & 177 \\
& 50 & $1.02 \pm 0.53$ & 8 & & $1.34 \pm 0.34$ & 11 \\
\hline
\end{tabular}

$2 \times 10^{5} \mathrm{MNC}$ were cultured with different interferon concentrations and with or without dexamethasone for 7-8 d. Cultures were refed by $1 / 2$ medium change at day 4 with addition of relevant reagents. Data represent mean of specific degradation of ${ }^{125}$ I-Man-BSA measured over $2 \mathrm{~d}$. 
Table $V$. Effects of $\gamma$-Ifn and Dexamethasone on Monocyte PA*

\begin{tabular}{llllll}
\hline & \multicolumn{5}{l}{ Ifn (U/ml) } \\
\cline { 3 - 6 }$\gamma$-Ifn & Dose & 0 & 0.25 & 2.5 & 25 \\
\hline S.L. $(38$ h) & Dex $^{-}$ & 12 & ND & 28 & 59 \\
J.C. $(25$ h) & Dex $^{-}$ & 29 & 43 & 46 & 49 \\
& Dex $^{+}$ & 27 & 28 & 32 & 50 \\
P.T.A. $(27$ h) & Dex $^{-}$ & 55 & 74 & 79 & ND \\
& Dex $^{+}$ & 55 & 35 & 44 & ND \\
I.W. $(62$ h) & Dex $^{-}$ & 32 & 40 & 40 & 53 \\
& Dex $^{+}$ & 22 & 20 & 17 & 33 \\
& & & & & \\
\hline
\end{tabular}

* $2 \times 10^{5}$ monocytes were cultured on ${ }^{125} \mathrm{I}$-fibrin in RPMI plus $5 \%$ autologous serum for different periods shown with indicated concentrations of Ifn and/or dexamethasone, at $5 \times 10^{-8} \mathrm{M}$. Results show cell-dependent fibrinolysis as a percentage of total trypsin-releasable radioactivity. Cell-independent release ranged between 2 and $7 \%$, depending on the duration of the experiment. Preliminary experiments showed that fibrinolysis under these conditions depended on the presence of plasminogen. Results are average of duplicate assays. ND, not done.

shown), $\alpha$-Ifn also enhanced fibrinolytic activity, and this increase could be prevented by dexamethasone.

Table VI shows that human $\mathbf{M} \phi$ cultivated for $8 \mathrm{~d}$ expressed respiratory burst activity when challenged with phorbol myristate acetate (PMA) or zymosan. Ifn- $\gamma$ at relatively high concentrations enhanced the response to both stimuli 2-4-fold

Table VI. Effect of $\gamma$-Ifn and Dexamethasone on Macrophage RB Activity

\begin{tabular}{|c|c|c|c|c|}
\hline \multicolumn{5}{|c|}{ HMP shunt activity (cpm/10 $\mathrm{M} \phi)$} \\
\hline & Basal & & PMA & Zymosan \\
\hline No treatment & 157 & & 1,585 & 853 \\
\hline$\gamma$-Ifn $50 \mathrm{U} / \mathrm{ml}$ & 169 & & 2,142 & 1,255 \\
\hline$\gamma$-Ifn $250 \mathrm{U} / \mathrm{ml}$ & 206 & & 3,029 & 4,086 \\
\hline \multirow[t]{3}{*}{$\gamma$-Ifn $500 \mathrm{U} / \mathrm{ml}$} & 350 & & 3,349 & 3,129 \\
\hline & & \multicolumn{3}{|l|}{ PMA } \\
\hline & & No Ifn & & $\gamma-\mathrm{Ifn} 250 \mathrm{U} / \mathrm{ml}$ \\
\hline No dexamethasone & & 1,460 & & 3,112 \\
\hline $\operatorname{Dex} 1 \times 10^{-8} \mathrm{M}$ & & ND & & 3,690 \\
\hline Dex $5 \times 10^{-8} \mathrm{M}$ & & 1,694 & & 3,596 \\
\hline Dex $1 \times 10^{-7} \mathrm{M}$ & & 1,526 & & 4,554 \\
\hline
\end{tabular}

$10^{6}$ adherent monocytes were cultured in RPMI plus $10 \%$ autologous serum for $\mathbf{8 d}$ in the presence of $\gamma$-Ifn or dexamethasone. Dexamethasone was added again every alternate day. Medium with or without additives was replaced on day 3 . After $8 \mathrm{~d}$, cultures were washed twice with warm serum-free medium and the HMP shunt assay was started by adding $0.75 \mu \mathrm{Ci}{ }^{14} \mathrm{Cl}$-glucose/well in $500 \mu \mathrm{l}$ reaction buffer (Krebs-Ringers phosphate buffer with glucose and calcium) with or without PMA $(100 \mathrm{ng} / \mathrm{ml})$ or zymosan $(100 \mu \mathrm{g} / \mathrm{ml})$ for $1 \mathrm{~h}$ at $37^{\circ} \mathrm{C}$. Radioactivity released into the medium was determined as described (17). Background ${ }^{14} \mathrm{C}$ release was between 60 and $90 \mathrm{cpm}$; adherent protein for all groups was 35-50 $\mu \mathrm{g}$ (average $42 \mu \mathrm{g} /$ well) irrespective of treatment. Results show average of replicate assays for one experiment each, representative of four independent experiments. as reported by others (3). Continuous exposure to dexamethasone, $1-10 \times 10^{-8} \mathrm{M}$, did not prevent this effect (Table VI B and unpublished results).

\section{Discussion}

Our studies show that it is possible to activate human monocytes/M $\phi$ from blood under defined conditions in vitro, that the MFR is an excellent marker of the effects of immune lymphocytes and interferons on $\mathbf{M} \phi$, and that the phenotype of $\mathbf{M} \phi$ can be selectively modulated by opposing actions of lymphokines and dexamethasone. Freshly isolated monocytes from healthy individuals display features of activated $\mathbf{M} \phi$ such as enhanced spreading (19), DR (Ia) antigen expression (20), respiratory burst activity (21), and low levels of MFR. During maturation in culture promoted by unidentified factors in human serum, monocytes lose much of their RB responsiveness (21) and acquire high levels of MFR, which are characteristic of nonactivated $\mathrm{M} \phi$ (22). However, exposure to products of immunologically stimulated lymphocytes and interferon enhances monocyte M $\phi$ RB $(3,21)$, PA $(23,24)$, antimicrobial (25) and cytocidal (26) activities, alters the expression of FcR (27) and class II antigens (28), and reduces receptor-mediated endocytosis via MFR. These lymphokines are therefore able to prevent or reverse one pathway of monocyte differentiation and induce another without necessarily influencing cell proliferation. Both $\gamma$ - and $\alpha$-Ifn inhibit the growth of myelomonocytic progenitors and leukemic cells and induce various markers of $\mathbf{M} \phi$ differentiation (29).

Down-regulation of the MFR provides a sensitive, $\mathbf{M} \phi$ specific, and selective marker of immune activation and can be readily assayed in the presence of other cells. Failure of activated $\mathrm{M} \phi$ to degrade ${ }^{125} \mathrm{I}-\mathrm{Man}-\mathrm{BSA}$ is accompanied by low binding and uptake of the ligand, which indicates a lack of receptor activity at the plasma membrane. This could be due to inhibition of MFR synthesis or enhanced turnover of receptors.

We have correlated MFR decrease with enhanced PA and RB activities, which are other markers of human $\mathbf{M} \phi$ "activation." One advantage of the MFR marker is that the effects of modulating agents on its expression are striking, since monocytes lack MFR activity initially. In contrast, fluctuations in RB activity, induced by lymphokines, are harder to detect because of variable background levels in untreated cells (21). MFR changes can be detected within 2-3 d; altered expression of FcR occurs earlier (27), but this receptor is not unique to $M \phi$. Enhanced fibrinolysis occurs within $1 \mathrm{~d}$, but is not specific for $\mathbf{M} \phi$.

We have shown that interferons can account for downregulation of M $\phi$ MFR by lymphocyte products, as demonstrated recently for other assays of $\mathbf{M} \phi$ activation (3-5), although additional mediators are not excluded. Both $\gamma$ - and $\alpha$-Ifn were potent inhibitors of MFR expression and specific antibodies selectively blocked the action of $\alpha$-Ifn. Interferons acted on highly purified monocytes/M $\phi$ targets and the effect on MFR was not due to direct inhibition of receptor activity. Ifn action was prolonged in the continuous presence of the agent and could be reversed by washout. It has been reported that $\gamma$-Ifn induces M $\phi$ RB and cidal activities $(3,26)$ far more effectively than $\alpha$-Ifn, which does enhance PA release (24) and FcR expression $(30,31)$. Apart from possible differences in the nature of the Ifn preparations and in the particular marker studied, different $\mathbf{M} \phi$ targets could also vary in their response 
to Ifn. We have noted that only murine $\gamma$ and not $\alpha$-/ $\beta$-Ifn decreases MFR activity in mouse $\mathbf{M} \phi$ targets (Ezekowitz, R. A. B., unpublished observations). $\gamma$ - and $\alpha$-Ifn are products of distinct genes (32), differ immunologically (33), bind to distinct cellular receptors $(34,35)$, and induce unique as well as common polypeptides (36) in non-M $\phi$ targets. Our studies show that both Ifn can enhance or decrease particular target cell functions. The selective antagonism observed by dexamethasone in some experiments could indicate distinct effects of $\gamma$ - and $\alpha$-Ifn on some human M $\phi$ functions.

The ability of dexamethasone to counteract the decrease in MFR activity induced by antigen and $\boldsymbol{\gamma}$-Ifn is of considerable interest. Enhanced fibrinolysis, a marker which is not restricted to immune activation of $\mathbf{M} \phi$ (12), was also antagonized by dexamethasone, a well-known inhibitor of $\mathbf{M} \phi$ PA production $(37,38)$. We confirmed that $\gamma$-Ifn also enhanced RB activity in human monocytes/M $\phi$ (3), but this effect could not be prevented by dexamethasone, unlike other studies in which hydrocortisone inhibited $\mathrm{H}_{2} \mathrm{O}_{2}$ release by lymphokine-treated human $\mathrm{M} \phi$ at relatively high concentrations (21). The difference between effects of glucocorticoids on RB activity and other M $\phi$ activation markers could be related to the high levels of oxidase activity present in human monocytes at the time of isolation.

In addition to complex direct actions on $\mathrm{M} \phi$ (38), glucocorticoids act on lymphocytes and their interaction with $\mathbf{M} \phi$ (reviewed in 39). Dexamethasone inhibits production of lymphokines (39) and their action on $\mathbf{M} \phi$ (40). In our studies, dexamethasone antagonized the action of $\gamma$-Ifn on $\mathbf{M} \phi$ in the absence of lymphocytes in a concentration-dependent manner. Glucocorticoids induce the release from $\mathbf{M} \phi$ of macrocortin, a phospholipase $A 2$ inhibitor (41), and reduced prostaglandin generation could influence Ifn action on $M \phi(42)$. Dexamethasone may also enhance the expression of MFR directly (43), as observed in some of our experiments in the absence of lymphokine, perhaps due to increased synthesis of plasma membrane receptors (44).

The system described can be used to study the role of antigen, lymphocyte subpopulations, and humoral factors in activation of human $\mathbf{M} \phi$. We have noted striking defects in $\mathbf{M} \phi$ activation in some patients infected with $M$. tuberculosis or atypical mycobacteria. Expression of M $\phi$ MFR provides a sensitive indicator to study the balance between cellular immunity and immunosuppression and control of $\mathbf{M} \phi$ activity by activating and suppressive lymphocyte products (45) and pharmacologic agents.

\section{Acknowledgments}

We thank Dr. C. Entwistle and his staff at the Oxford Regional BTS for organising and bleeding volunteers, and Mrs. Pam Woodward for typing the manuscript.

This work was supported by the Medical Research Council, United Kingdom.

\section{References}

1. Cohn, Z. A. 1978. Activation of mononuclear phagocytes: fact fancy and future. J. Immunol. 121:813-816.

2. Ezekowitz, R. A. B., and S. Gordon. 1984. Alterations of surface properties by macrophage activation. Expression of receptors for $\mathrm{Fc}$ and mannose-terminal glycoproteins and differentiation antigens. In Contemporary Topics in Immunobiology. Vol. 13. D. O. Adams and
M. G. Hanna, Jr., editors. Plenum Publishing Corp., New York. 3356.

3. Nathan, C. F., H. W. Murray, M. E. Wiebe, and B. Y. Rubin. 1983. Identification of Interferon- $\gamma$ as the lymphokine that activates human macrophage oxidative metabolism and antimicrobial activity. J. Exp. Med. 158:670-689.

4. Schreiber, R. D., J. L. Pace, S. W. Russell, A. Altman, and D. H. Katz. 1983. Macrophage-activating factor produced by a T cell hybridoma: physiochemical and biosynthetic resemblance to $\gamma$-interferon. J. Immunol. 131:826-832.

5. Steeg, P. S., R. N. Moore, H. M. Johnson, and J. J. Oppenheim. 1982. Regulation of murine macrophage Ia antigen expression by a lymphokine with immune interferon activity. J. Exp. Med. 156:17801793.

6. Ezekowitz, R. A. B., and S. Gordon. 1982: Down-regulation of mannosyl receptor mediated endocytosis and antigen F4/80 in Bacillus Calmette-Guerin-activated mouse macrophages. Role of $\mathrm{T}$ lymphocytes and lymphokines. J. Exp. Med. 155:1623-1637.

7. Gray, P. W., D. W. Leung, D. Pennica, E. Yelverton, R. Najarian, C. C. Simonsen, R. Derynck, P. J. Sherwood, D. M. Wallace, S. L. Berger, A. D. Levinson, and D. V. Goeddel. 1982. Expression of human immune interferon cDNA in $E$. coli and monkey cells. Nature (Lond.). 295:503-508.

8. Fantes, K. H., C. J. Burman, G. D. Ball, M. D. Johnston, and N. B. Finter. 1980. Production, purification and properties of human lymphoblastoid interferon. In Biochemical Characterization of Lymphokines. A. L. de Weck, F. Kristensen, and M. Landy, editors. Academic Press, New York and London. 343-351.

9. Allan, G., K. H. Fantes, D. C. Burke, and J. Morser. 1982. Analysis and purification of human lymphoblastoid (Namalva) interferon using a monoclonal antibody. J. Gen. Virol. 63:207-212.

10. Stahl, P. D., J. S. Rodman, M. J. Miller, and P. H. Schlesinger. 1978. Evidence for receptor-mediated binding of glycoproteins, glycoconjugates and lysosomal glycosidases by alveolar macrophages. Proc. Natl. Acad. Sci. USA. 75:1399-1403:

11. Greenwood, F. C., W. M. Hunter, and J. S. Glover. 1963. The preparation of ${ }^{131} \mathrm{I}$-labelled human growth hormone of high specific radioactivity. Biochem. J. 89:114-123.

12. Gordon, S. 1979. Assays for macrophage activation and secretion products. In Handbook of Experimental Immunology. D. M. Weir, editor. 3rd ed. Blackwell Scientific Publications, Oxford. 33.1-33.14.

13. Li, C. Y., K. W. Lam, and L. T. Yam. 1973. Esterases in human leukocytes. J. Histochem. Cytochem. 21:1-12.

14. Lin, H.-S., and S. Gordon. 1979. Secretion of plasminogen activator by bone marrow-derived mononuclear phagocytes and its enhancement by colony-stimulating factor. J. Exp. Med. 150:231-245.

15. Stahl, P., and S. Gordon. 1982. Expression of a mannosylfucosyl receptor by macrophages and their hybrids. J. Cell Biol. 93:4956.

16. Oppenheim, J. J., and D. L. Rosenstreich. 1976. Lymphocyte transformation: utilization of automatic harvesters. In In Vitro Methods in Cell-mediated and Tumor Immunity. B. R. Bloom and J. R. David, editors. Academic Press, Inc., New York. 573-585.

17. Berton, G., and S. Gordon. 1983. Superoxide release by peritoneal and bone marrow-derived mouse macrophages. Modulation by adherence and cell activation. Immunology. 49:693-704.

18. Shepherd, V. L., E. J. Campbell, R. M. Senior, and P. D. Stahl. 1982. Characterization of mannose-fucose receptor on human mononuclear phagocytes. J. Reticuloendothel. Soc. 32:423-431.

19. Gotze, O., C. Bianco, J. S. Sundsmo, and Z. A. Cohn. 1980. The stimulation of mononuclear phagocytes by components of the classical and the alternative pathways of complement activation. In Mononuclear Phagocytes. Functional Aspects. R. van Furth, editor. Martinus Nijhoff, The Hague. 1421-1442.

20. Mason, R., J. Austyn, F. Brodsky, and S. Gordon. 1982. Monoclonal antimacrophage antibodies: human pulmonary macrophages express HLA-DRW (Ia-like) antigens. Am. Rev. Resp. Dis. 125:586-593. 
21. Nakagawara, A., N. M. de Santis, N. Nogueira, and C. F. Nathan. 1982. Lymphokines enhance the capacity of human monocytes to secrete reactive oxygen intermediates. J. Clin. Invest. 70:1042-1048.

22. Ezekowitz, R. A. B., J. Austyn, P. D. Stahl, and S. Gordon. 1981. Surface properties of Bacillus Calmette-Guerin-activated mouse macrophages. Reduced expression of mannose specific endocytosis. Fc receptors and antigen F4/80 accompanies induction of Ia. J. Exp. Med. 154:60-76.

23. Greineder, D. J., K. J. Connorton, and J. R. David. 1979. Plasminogen activator production by human monocytes. I. Enhancement by activated lymphocytes and lymphocyte products. J. Immunol. 123:2808-2813.

24. Hovi, T., O. Saksela, and A. Vaheri. 1981. Increased secretion of plasminogen activator by human macrophages after exposure to leukocyte interferon. FEBS (Feb. Eur. Biochem. Soc.) Lett. 129:233236.

25. Murray, H. W., and Z. A. Cohn. 1980. Macrophage oxygendependent antimicrobial activity. III. Enhanced oxidative metabolism as an expression of macrophage activation. J. Exp. Med. 152:15961609.

26. Le, J., W. Prensky, Y. K. Yip, Z. Chang, T. Hoffman, H. C. Stevenson, I. Balazs, J. R. Sadlik, and J. Vilcek. 1983. Activation of human monocyte cytotoxicity by natural and recombinant immune interferon. J. Immunol. 131:2821-2826.

27. Perussia, B., E. T. Dayton, R. Lazarus, V. Fanning, and G. G. Trinchieri. 1983. Immune interferon induces the receptor for monomeric IgG1 on human monocytic and myeloid cells. J. Exp. Med. 158:10921113.

28. Basham, T. Y., and T. C. Merigan. 1983. Recombinant interferon gamma increases HLA-DR synthesis and expression. $J$. Immunol. 130:1492-1494.

29. Perussia, B., E. T. Dayton, V. Fanning, P. Thiagarajan, J. Hoxie, and G. Trinchieri. 1983. Immune interferon and leukocyteconditioned medium induce normal and leukemic myeloid cells to differentiate along the monocytic pathway. J. Exp. Med. 158:20582080.

30. Rhodes, J., and P. Stokes. 1982. Interferon-induced changes in monocyte membrane: inhibition by retinol and retinoic acid. Immunology. 45:531-536.

31. Rhodes, J., D. H. Jones, and N. M. Bleehen. 1983. Increased expression of human monocytes HLA-DR antigens and receptors in response to human interferon in vivo. Clin. Exp. Immunol. 53:739743.

32. Toy, T. L. 1983. The interferons (Review). Clin. Exp. Immunol. 54:1-13.

33. de Ley, M., J. van Damme, H. Claeys, H. Weeming, J. W.
Heine, A. Billian, C. Vermylen, and P. de Somer. 1980. Interferon induced in human leukocytes by mitogens: production partial purification and characterization. Eur. J. Immunol. 10:877-883.

34. Branca, A. A., and C. Baglioni. 1981. Evidence that types I and II interferons have different receptors. Nature (Lond.). 294:768770 .

35. Joslin, A. R., F. H. Sarkar, and S. L. Gupta. 1982. Interferon receptors. Crosslinking of human leukocyte interferon $\alpha_{2}$ to its receptor on human cells. J. Biol. Chem. 257:13884-13887.

36. Weil, J., C. J. Epstein, L. B. Epstein, J. J. Sedman, J. L. Sabran, and S. E. Grossberg. 1983. A unique set of polypeptides induced by $\gamma$-interferon in addition to those induced in common with $\alpha$ and $\beta$ interferons. Nature (Lond.). 301:437-439.

37. Vassali, J.-D, J. Hamilton, and E. Reich. 1976. Macrophage plasminogen activator: modulation of enzyme production by antiinflammatory steroids, mitotic inhibitors and cyclic nucleotides. Cell. 8: 271-281.

38. Werb, Z. 1980 . Hormone receptors and hormonal regulation of macrophage physiological functions. In Mononuclear Phagocytes. Functional Aspects. R. van Furth, editor. Martinus Nijhoff, The Hague. 809-835.

39. Fairchild, S. S., and R. I. Mishell. 1982. Glucocorticosteroid response modifying factors. Lymphokine Research. 1:113-120.

40. Barlow, J. E., and A. S. Rosenthal. 1973. Glucocorticoid suppression of macrophage migration inhibitory factor. J. Exp. Med. 137:1031-1041.

41. Flower, R. J., J. N. Wood, and L. Parente. 1984. Macrocortin and the mechanism of action of the glucocorticoids. In Advances in Inflammation Research. Vol. 1. T. Otterness, R. Capetola, and S. Wong, editors. Raven Press, New York. 61-70.

42. Koren, H. S., S. J. Anderson, D. G. Fischer, C. S. Copeland, and P. J. Jensen. 1981. Regulation of human natural killing. I. The role of monocytes, interferon, and prostaglandins. J. Immunol. 127: 2007-2013.

43. Konish, M. G., D. Thomasson, and P. D. Stahl. 1982. Expression of mannose receptor by rat bone marrow derived macrophages: modulation of receptor activity by lymphokines and glucocorticoids. Fed. Proc. 41:3764 (Abstr.)

44. Salhanick, A. I., M. N. Krupp, and J. M. Amatruda. 1983. Dexamethasone stimulates insulin receptor synthesis in cultured rat hepatocytes. J. Biol. Chem. 258:14130-14135.

45. Fogelman, A. M., J. Seager, J. E. Groopman, J. A. Berliner, M. E. Haberland, P. A. Edwards, and D. W. Golde. 1983. Lymphokines secreted by an established lymphocyte line modulate receptor mediated endocytosis in macrophages derived from human monocytes. J. Immunol. 131:2368-2373. 